\title{
Purification and Uniqueness of Quantum Gibbs States
}

\author{
Taku Matsui ${ }^{\star}$ \\ Institute for Theoretical Physics, University of Leuven, Belgium
}

Received: 24 May 1993

Abstract: We give a new condition for uniqueness of Gibbs states of quantum spin models on lattices.

\section{Introduction}

In this paper, we propose a new method for proving uniqueness of Gibbs states of quantum spin models on lattices. Our method is based on the ergodicity of Markov semigroups (semigroups of completely positive maps on $C^{*}$-algebras). This is a standard method in classical spin models, but has never been developed in quantum cases. Our motivation was to find a quantum analogue of Dobrushin's uniqueness condition for Gibbs measure ([4 and 5]), though the condition we obtained in this paper is still a perturbation theory near the infinite temperature.

In our opinion, we encounter two types of difficulties originating from the noncommutativity when considering the uniqueness theorem for quantum models.

(i) The problem of entire analyticity: Let $\alpha_{t}(Q)$ be the time evolution of a local observable $\mathbf{Q}$, formally written as $\alpha_{t}(Q)=e^{i t H} Q e^{-i t H}$, where $\mathrm{H}$ is the infinite volume Hamiltonian. The notion of quantum DLR equation was first introduced in [1]. The DLR equation for a quantum state $\varphi$ is

$$
\varphi(Q)=\operatorname{tr}_{I} \otimes \psi\left(\Lambda_{I}^{*} Q \Lambda_{I}\right),
$$

where $\operatorname{tr}_{I}$ is the normalized trace on the volume $I, \psi$ is a (possibly non-normalized) positive linear functional of the observables supported outside $I$, and $\Lambda_{I}$ is an operator given by the help of the relative modular operator of the modular theory of von Neumann algebras. $\Lambda_{I}$ has a formal expression,

$$
\Lambda_{I}=\exp \left(-1 / 2\left(H-H_{I}\right)\right) \exp (1 / 2 H),
$$

where $H_{I}$ is the finite Hamiltonian on the volume I with boundary terms.

\footnotetext{
* Research fellow of Canon Foundation in Europe, on leave from Tokyo Metropolitan University, Hachioji, 192-03 Japan
} 
The fact that the operator $\Lambda_{I}$ is defined dependent of the choice of the state is an obstacle for considering uniqueness, which is absent in classical spin models. If the time evolution $\alpha_{t}(Q)$ as a function of $t$ is extendible to an entire analytic function on the complex plane, $\Lambda_{I}$ is defined as an element of the $C^{*}$-algebra independent of the state chosen and this problem does not emerge.

(ii) Nature of Markov property of quantum states: In classical spin models, the local Markov property implies that the measure concerned is Gibbsian while non-commutative systems seem to have no analogous result. Alternatively saying, in classical spin systems, the Gibbs distribution is a superposition of the finite volume states for the various boundary conditions while we have not yet known a good notion of boundary condition useful for the quantum DLR equation. The quantum Gibbs state is not characterized by conditional expectations. It is known that any completely positive map reversible with respect to (satisfying the detailed balance condition for) a KMS state (= Gibbs state) commutes with the time evolution (modular automorphism). (See Lemma 2 of [3] and Proposition 1 of [6].) This is a constraint to construct Markov semigroups for which reversible states are Gibbs states of a given interaction.

We have not yet obtained satisfactory results on the first problem of analyticity in this paper. As for the second problem, our strategy is as follows. The starting point is that we do not consider states of the algebra of local observables $\mathscr{A}$ but those of the tensor product $\mathscr{A} \otimes \mathscr{A}$. By use of the modular conjugation operator, we can associate a canonical pure state of $\mathscr{A} \otimes \mathscr{A}$ with each Gibbs state of pure phase. This procedure is called purification. Once we go to the purification, the constraint from the modular theory of von Neumann algebras disappears. Next we introduce Hamiltonians for which any ground state is necessarily the purification of a Gibbs state. We also construct a Markov semigroup on $\mathscr{A} \otimes \mathscr{A}$ for which the purification of any Gibbs state is reversible. (The reversibility is defined in a standard way as in Definition 3 of this paper.)

Thus, our proposal is simple. Consider the purification of quantum Gibbs states and prove the ergodicity of the Markov semigroup for which the invariant state is a purified Gibbs state. Our condition for unicity of the invariant state of our Markov semigroup then follows from the idea of [7].

Both Markov semigroups and Hamiltonians considered in this paper are not canonically defined and there lies a room of modification of the construction of the semigroup and the Hamiltonian. We are expecting the improvement of results at this step. In the classical spin language, this corresponds to the point that we have still some choices for spin flip rate functions in interacting particle systems.

In Sect. 2 the basic idea will be explained. Our main result is Proposition 2. In Sect. 3 , we give estimates which imply that our unicity condition is valid for the high temperature short range interactions.

\section{Purification of KMS States}

In what follows, we consider the spin $1 / 2$ system. The higher spin case may be treated analogously, for example, in [7] we considered the $Z_{N}$ symmetric systems. There are various ways to handle higher spin models depending on the symmetry of Hamiltonians. 
We use the $C^{*}$-algebraic framework of quantum spin models. (See [2].) Let $\mathscr{A}$ be the $C^{*}$-algebra of quantum observables for spin $1 / 2$ on the $d$ dimensional regular lattice $Z^{d}$. More precisely, $\mathscr{A}$ is the $C^{*}$-algebra completion of the infinite tensor product of the algebra of 2 by 2 matrices $M_{2}(C)$,

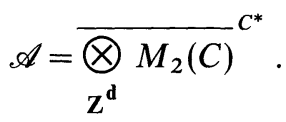

Each component of $\mathscr{A}$ is labeled by a point $j$ of the square lattice $\mathbf{Z}^{\mathbf{d}}$. Let $\mathbf{Q}$ be a 2-by-2 matrix. By $Q^{(j)}$ we denote an element of $\mathscr{A}$ with $\mathbf{Q}$ in the $j$ component and the identity in the other. We also denote the dense algebra generated by strictly local elements (polynomials of $Q^{(j)}$ by $\mathscr{A}_{\text {loc }}$ and for a subset $\Lambda$ of $\mathbf{Z}^{\mathrm{d}}, \mathscr{A}_{\Lambda}$ will be the subalgebra generated by $Q^{(j)}\left(j \in \Lambda, Q \in M_{2}(C)\right)$. The translation $\tau_{k}$ is an automorphism of $\mathscr{A}$ determined by $\tau_{k}\left(Q^{(j)}\right)=Q^{(j+k)}\left(k, j \in \mathbf{Z}^{\mathrm{d}}\right)$.

We regard $\mathscr{A}$ as the set of continuous functions on a non-commutative space. We will use a calculus on this non-commutative space. Set

$$
X=Z^{d} \times\{x, z\} .
$$

By $\sigma_{\alpha}^{(j)}(\alpha=x, y, z)$ we denote the Pauli spin matrix on the site $j$. For $b=(\alpha, j)$ in $X$ and $\mathbf{Q}$ in $\mathscr{A}$, we define the differential $\delta_{b}$ by the equation,

$$
\delta_{b}(Q)=1 / 2\left(\sigma_{\alpha}^{(j)} Q \sigma_{\alpha}^{(j)}-Q\right) .
$$

Then it is easy to check that

$$
\begin{aligned}
\delta_{b}^{2}(Q) & =-\delta_{b}(Q), \\
\left\|\delta_{b}(Q)\right\| & =1 / 2\left\|\left[\sigma_{b}^{(j)}, Q\right]\right\| .
\end{aligned}
$$

We also consider $\mathrm{n}$ times differentiable elements, smooth elements etc. define

Let $F_{n}$ be the set of subsets with $n$ elements in $X$. For $A=\left\{k_{1}, k_{2} \ldots k_{n}\right\} \in F_{n}$, we

$$
\delta_{A}(Q)=\delta_{k_{1}} \delta_{k_{2}} \delta_{k_{1}} \ldots \delta_{k_{n}}(Q)
$$

We set

$$
\|Q\|=|\|Q\||_{0}
$$

For a non-negative integer $n$,

$$
\left.\|Q\|\right|_{n}=\sum_{A \in F_{n}}\left\|\delta_{A}(Q)\right\|
$$

An $n$ times differentiable element should be $\mathbf{Q}$ such that $|\|Q\||_{n}$ is finite, so we set

$$
\begin{aligned}
C^{n}(\mathscr{A}) & =\left\{\left.Q \in \mathscr{A}||\|Q\|\right|_{n}<\infty(0 \leqq m \leqq n)\right\}, \\
C_{\lambda}^{\infty}(\mathscr{A}) & =\left\{\left.Q \in \mathscr{A}\left|\sup _{n} \lambda^{n}\right|\|Q\|\right|_{n}<\infty\right\} .
\end{aligned}
$$

If $\mathbf{Q}$ is a local element, $|\|Q\||_{n}$ vanishes for $n$ sufficiently large.

Lemma 1. Let $Q$ be an element of $C_{\lambda}^{\infty}(\mathscr{A})$ with $\lambda>1$. Then $Q$ can be written in the following form:

$$
Q=\sum_{A, B} C_{A B}(Q) \prod_{j \in A} \sigma_{z}^{(j)} \prod_{j \in B} \sigma_{x}^{(j)}
$$


where the sum is taken over all the finite sets of $Z^{d}$ and $C_{A B}(Q)$ is a complex number determined by

$$
C_{A B}(Q)=\operatorname{tr}\left(\prod_{j \in B} \sigma_{x}^{(j)} \prod_{j \in A} \sigma_{z}^{(j)} Q\right) .
$$

The sum of (11) is convergent in the norm of the $C^{*}$-algebra $\mathscr{A}$ and

$$
\sum_{A, B}|A \cup B|^{k}\left|C_{A B}(Q)\right|<\infty
$$

for any positive $k$.

Proof. Suppose that $l \in A, l \notin B$. By use of anticommutativity of Pauli matrices and $1=1 / 2\left\{1+\sigma_{x}^{(l)^{2}}\right\}$, we have

$$
\begin{aligned}
1 / 2 & \left.\left\{\operatorname{tr}\left(\prod_{j \in B} \sigma_{x}^{(j)} \prod_{j \in A} \sigma_{z}^{(j)} Q\right)+\operatorname{tr}\left(\prod_{j \in B} \sigma_{x}^{(j)} \prod_{j \in A} \sigma_{z}^{(j)} \sigma_{x}^{(l)}\right)^{2} Q\right)\right\} \\
& =1 / 2 \operatorname{tr}\left(\prod_{j \in B} \sigma_{x}^{(j)} \prod_{j \in A} \sigma_{z}^{(j)}\left(-\sigma_{x}^{(l)} Q \sigma_{x}^{(l)}+Q\right)\right) \\
& =-\operatorname{tr}\left(\prod_{j \in B} \sigma_{x}^{(j)} \prod_{j \in A} \sigma_{z}^{(j)} \delta_{(l, x)}(Q)\right) .
\end{aligned}
$$

Thus we have

$$
C_{A B}(Q)=-\operatorname{tr}\left(\prod_{j \in B} \sigma_{x}^{(j)} \prod_{j \in A} \sigma_{z}^{(j)} \delta_{(l, x)}(Q)\right) .
$$

Other cases $l \notin A l \in B$, or $l \in A, l \in B$ can be treated in the same way, and we arrive at the estimate,

$$
\left|C_{A B}(Q)\right| \leqq\left\|\delta_{\left(j_{1}, x\right)} \delta_{\left(j_{1}, x\right)} \ldots \delta_{\left(j_{n}, x\right)} \delta_{\left(i_{1}, z\right)} \ldots \delta_{\left(i_{m}, z\right)}(Q)\right\|,
$$

where the elements of the sets $A$ and $B$ are labeled as follows:

$$
A=\left\{j_{1}, j_{2}, \ldots, j_{n}\right\} B-A \cup B=\left\{i_{1}, i_{2}, \ldots, i_{m}\right\} .
$$

As a consequence,

$$
\begin{aligned}
\sum_{A, B}|A \cup B|^{k}\left|C_{A B}(Q)\right| & \leqq \sum_{n} n^{k} \lambda^{-n}\left(\sup _{m} \lambda^{m}|\|Q\||_{m}\right) \\
& <\frac{k !}{(1-\lambda)^{k}}\left(\sup _{m} \lambda^{m}|\|Q\||_{m}\right)<\infty
\end{aligned}
$$

Next we consider the time evolution. We will always consider the translationally invariant interactions, but the non-translational invariance causes no trouble.

Let $h$ be a selfadjoint element of $\mathscr{A}$ determined by

$$
h=\sum_{A, B} h_{A B} \prod_{j \in A} \sigma_{z}^{(j)} \prod_{k \in B} \sigma_{x}^{(k)}
$$


The selfadjointness implies

$$
\overline{h_{A B}}=(-1)^{|A \cup B|} h_{A B},
$$

where $|A \cup B|$ is the number of points in $A \cup B$. The (infinite volume) Hamiltonian is

$$
H=\sum_{j \in Z^{d}} h_{j}, \quad h_{j}=\tau_{j}(h) .
$$

Consider the derivation $\delta^{H}$ determined by

$$
i[H, Q]=\delta^{H}(Q) .
$$

Using the ideas of [7] we can prove the following results. If the following condition is satisfied, the derivation $\delta^{H}$ is well-defined on $C^{1}(\mathscr{A})$,

$$
\sum_{A, B}\left|h_{A B}\right||A \cup B|<\infty .
$$

Moreover, the interaction satisfying the decay condition,

$$
\sum_{A, B}\left|h_{A B}\right||A \cup B|^{2}<\infty
$$

gives rise to the time evolution $\alpha_{t}$,

$$
\alpha_{t}(Q)=e^{i t H} Q e^{-i t H} .
$$

Strictly speaking, the closure of the derivation $\delta^{H}$ is the generator of the 1parameter group of automorphisms $\alpha_{t}$ of $\mathscr{A} \cdot \alpha_{t}$ leaves $C^{1}(\mathscr{A})$ globally invariant and for $\mathbf{Q}$ in $C^{1}(\mathscr{A})$,

$$
\frac{d}{d t} \alpha_{t}(Q)=\alpha_{t}\left(\delta^{H}(Q)\right) .
$$

(See [7].) However, it is not yet known whether the set $\mathscr{A}_{\text {loc }}$ of (strictly) local observables is a core for the generator of $\alpha_{t}$. It may happen that $\alpha_{t}(Q)$ does not admit a complex holomorphic extension as a function of $t$. As the KMS boundary condition of Gibbs states is involved with complex holomorphic extension of the time dependent correlation functions, this situation is troublesome. So we assume a stronger decay condition for the interaction from now on. Namely,

$$
\sum_{A, B}\left|h_{A B}\right||A \cup B| \exp (\varepsilon|A \cup B|)<\infty
$$

for a positive $\varepsilon$.

For the Hamiltonian satisfying (23), the notions of Gibbs states and KMS states are equivalent. The Gibbs state is also characterised by the variational principle for free energy. These results were first established in the 1970's in [1] and in a subsequent work of Araki. (See the historical explanation and the references in [2].) Here we just recall the definition of the KMS state.

A state $\varphi$ of $\mathscr{A}$ is a $\beta$ KMS state for $\alpha_{t}$ if

$$
\varphi(A B)=\varphi\left(\alpha_{-i \beta / 2}(B) \alpha_{i \beta / 2}(A)\right)
$$

for any $A, B$ in a dense * subalgebra of entire analytic elements. 
Here $\beta$ is the inverse temperature. It is known that a KMS state is faithful (separating) in our situation as $\mathscr{A}$ is simple. (Corollary 5.3.9 of [2]) and the state for a pure phase is a factor state.

Definition 1. Let $\varphi$ be a faithful state of $\mathscr{A}$. Let $\{\pi, \Omega, \mathscr{H}\}$ be the GNS cyclic representation associated with the state $\varphi$. ( $\Omega$ is the cyclic separating vector for the von Neumann algebra $\pi(\mathscr{A})^{\prime \prime}$ on the Hilbert space $\mathscr{H}$.) Let $J$ be the modular conjugation operator associated with $\Omega$.

Let $\Gamma$ be the complex conjugation of $\mathscr{A}$. More precisely, $\Gamma$ is a conjugate linear map on $\mathscr{A}$ satisfying

$$
\Gamma\left(Q_{1} Q_{2}\right)=\Gamma\left(Q_{1}\right) \Gamma\left(Q_{2}\right)
$$

and

$$
\Gamma\left(\sigma_{\alpha}^{(j)}\right)=\sigma_{\alpha}^{(j)}(\alpha=x, z) \Gamma\left(\sigma_{y}^{(j)}\right)=-\sigma_{y}^{(j)} .
$$

Consider the $C^{*}$-algebra $\tilde{\mathscr{A}}=\mathscr{A} \otimes \mathscr{A}$ and the state $\tilde{\varphi}$ determined by

$$
\tilde{\varphi}(A \otimes B)=(\Omega, \pi(A) J \pi(\Gamma(B)) J \Omega),(A, B \in \mathscr{A}) \text {. }
$$

Then we call $\tilde{\varphi}$ the purification of $\varphi$.

We also introduce some notations for analysis on $\tilde{\mathscr{A}}$. Set

$$
\tilde{X}=\left\{\left(\alpha_{1}, \alpha_{2}, j\right) \mid \alpha_{1}, \alpha_{2}=0, x, y, z \quad j \in \mathbf{Z}^{\mathbf{d}}\right\}
$$

and $\sigma_{0}^{(k)}=1$.

Then for $\tilde{\alpha}=\left(\alpha_{1}, \alpha_{2}\right)$,

$$
\tilde{\delta}_{(\tilde{\alpha}, k)}(Q)=1 / 2\left\{\sigma_{\alpha_{1}}^{(k)} \otimes \sigma_{\alpha_{2}}^{(k)} Q \sigma_{\alpha_{1}}^{(k)} \otimes \sigma_{\alpha_{2}}^{(k)}-Q\right\} .
$$

Then $C^{n}(\tilde{\mathscr{A}})$ and $C_{\lambda}^{\infty}(\tilde{\mathscr{A}})$ are defined as in the same way for $\mathscr{A}$.

The next task is the characterization of states on $\tilde{\mathscr{A}}$ which are the purification of KMS states of $\mathscr{A}$. Here we make technical assumptions for the interaction.

Assumption 1. We assume that

(i) for any strictly local element $Q$ in $\mathscr{A}$, the time evolution $\alpha_{t}(Q)$ as a function of $t$ is extendible to a function $\alpha_{z}(Q)$ which is holomorphic on the strip $D_{\beta}=\left\{z \in \mathscr{C}|| z \mid<\frac{1}{2} \beta\right\}$ and continuous on the closure of $D_{\beta}$, and

(ii) for any $Q \in \mathscr{A}_{\mathrm{loc}}, \alpha_{z}(Q)$ is in $C_{\lambda}^{\infty}(\mathscr{A})$ with $\lambda>1$.

We believe that this assumption is valid for any finite range translationally invariant interaction and any inverse temperature $\beta$, but we are unable to prove it. This assumption holds trivially for classical finite range interactions (diagonal interactions). We will see its validity for the high temperatures in Sect. 4.

We now present a Hamiltonian $\widetilde{H}$ for which any ground state is the purification of a KMS state.

For $\alpha=x, y, z j \in \mathbf{Z}^{\mathrm{d}}$, we set

$$
\begin{gathered}
a_{\alpha}^{(j)}=1 / 2\left\{1-\sigma_{\alpha}^{(j)} \otimes \Gamma\left(\alpha_{\frac{i}{2} \beta}\left(\sigma_{\alpha}^{(j)}\right)\right)\right\} \in \tilde{\mathscr{A}}, \\
\widetilde{h_{j}}=1 / 2 \sum_{\alpha=x, y, z}\left\{a_{\alpha}^{(j)^{*}} a_{\alpha}^{(j)}+\left(a_{\alpha}^{(j)^{*}} a_{\alpha}^{(j)}\right)^{2}\right\}, \\
\tilde{H}=\sum_{j} \widetilde{h_{j}} .
\end{gathered}
$$



on $\tilde{\mathscr{A}}$.

If Assumption 1 is valid, the Hamiltonian $\tilde{H}$ yields a well-defined time evolution

Definition 2. We say that a state $\psi$ of $\tilde{\mathscr{A}}$ is a ground state for $\tilde{H}$ if

$$
\psi\left(a_{\alpha}^{(j)^{*}} a_{\alpha}^{(j)}\right)=0
$$

for any $\alpha=x, y, z$ and any $j \in \mathbf{Z}^{\mathbf{d}}$.

We next construct a Markov semigroup which we might call the stochastic Ising model on the non-commutative space $\mathscr{A}$. In this paper, a Markov semigroup always means a semigroup of completely positive maps on the $C^{*}$-algebra $\mathscr{A}$.

Set

$$
\begin{gathered}
D_{j}(\alpha, \gamma)=C_{j} \sigma_{\alpha}^{(j)} \otimes \Gamma\left(\alpha_{\frac{i}{2} \beta}\left(\sigma_{\gamma}^{(j)}\right)\right), \\
C_{j}=2\left(1-a_{x}^{(j)}\right)\left(1-a_{z}^{(j)}\right), \\
E_{j}(Q)=\frac{1}{16}\left\{4 \widetilde{h_{j}} Q \widetilde{h_{j}}+\sum_{\alpha \neq \gamma} D_{j}(\alpha, \gamma)^{*} Q D_{j}(\alpha, \gamma)\right\}, \\
L_{j}(Q)=E_{j}(Q)-\frac{1}{2}\left\{Q, E_{j}(1)\right\}, \\
L(Q)=\sum_{j \in \mathbf{Z}^{\mathbf{d}}} L_{j}(Q),
\end{gathered}
$$

$L(Q)$ is well-defined on $C^{1}(\tilde{\mathscr{A}})$. The closure of $L(Q)$ is the generator of a Markov semigroup $S_{t}$ due to Assumption 1 and results of [7],

$$
\frac{d}{d t} S_{t}(Q)=S_{t}(L(Q))
$$

Definition 3. We say that a state $\psi$ of $\tilde{\mathscr{A}}$ is reversible for $S_{t}$ if $\psi\left(A S_{t}(B)\right)=\psi\left(S_{t}(A) B\right)$ for any $A, B$ in $\tilde{\mathscr{A}}$.

The reversibility is often called the detailed balance condition.

Proposition 1. Suppose that Assumption 1 is valid for the interaction $h_{A, B}$.

(i) a state $\tilde{\psi}$ of $\tilde{\mathscr{A}}$ is the purification of a KMS state if and only if it is a ground state for $H$.

(ii) the purification $\tilde{\psi}$ of a KMS state $\psi$ is reversible for $S_{t}$. Furthermore, for the GNS representations $(\pi(), \Omega, \mathscr{H})$ associated with $\psi$ and the representation $\tilde{\pi}$ associated with $\tilde{\psi}$, we have

$$
\tilde{\pi}\left(S_{t}(Q)\right) \Omega=e^{-t \tilde{H}_{\psi}} \pi(Q) \Omega
$$

for $t>0$ and $Q \in \mathscr{A}$, where $\tilde{H}_{\psi}$ is a selfadjoint operator determined by the following limit (in the strong resolvent sense):

$$
\tilde{H}_{\psi}=\lim _{N} \sum_{|j| \leqq N} \tilde{\pi}\left(\tilde{h}_{j}\right) .
$$

Proof. All the statements are the consequences of the following identity:

$$
\begin{aligned}
\pi\left(\sigma_{\alpha}^{(j)}\right) \Omega & =J\left(J \Delta^{1 / 2}\right) \Delta^{-1 / 2} \pi\left(\sigma_{\alpha}^{(j)}\right) \Delta^{1 / 2} \Omega \\
& =J\left(\alpha_{-i \beta / 2}\left(\sigma_{\alpha}^{(j)}\right)\right)^{*} \Omega=\tilde{\pi}\left(1 \otimes \Gamma\left(\alpha_{i \beta / 2}\left(\sigma_{\alpha}^{(j)}\right)\right)\right) \Omega .
\end{aligned}
$$


Equation (38) implies

$$
\tilde{\pi}\left(a_{\alpha}\right) \Omega=0
$$

Here, we check only (ii).

Note that $\alpha_{\frac{1}{2} \beta}$ is an endomorphism from $\mathscr{A}_{\text {loc }}$. to $\mathscr{A}$. So we have

$$
\begin{gathered}
\left\{\alpha_{\frac{i}{2} \beta}\left(\sigma_{x}^{(j)}\right), \alpha_{\frac{i}{2} \beta}\left(\sigma_{z}^{(j)}\right)\right\}=0, \\
{\left[\sigma_{x}^{(j)} \otimes \alpha_{\frac{i}{2} \beta}\left(\sigma_{x}^{(j)}\right), \sigma_{z}^{(j)} \otimes \alpha_{\frac{i}{2} \beta}\left(\sigma_{z}^{(j)}\right)\right]=0,}
\end{gathered}
$$

and

$$
\left(1-a_{x}^{(j)}\right) \sigma_{y}^{(j)} \otimes \alpha_{\frac{1}{2} \beta}\left(\sigma_{x}^{(j)}\right)=\sigma_{y}^{(j)} \otimes \alpha_{\frac{i}{2} \beta}\left(\sigma_{x}^{(j)}\right) a_{x}^{(j)},
$$

etc. Then we can show

$$
\tilde{\pi}\left(D_{j}(\alpha, \gamma)\right) \Omega=0(\alpha, \gamma=x, y, z, \alpha \neq \gamma) .
$$

For example, if $\alpha=y, \gamma=x$,

$$
\tilde{\pi}\left(C_{j} \sigma_{y}^{(j)} \otimes \alpha_{\frac{i}{2} \beta}\left(\sigma_{x}^{(j)}\right)\right) \Omega=\tilde{\pi}\left(\left(1-a_{z}^{(j)}\right) \sigma_{y}^{(j)} \otimes \alpha_{\frac{i}{2} \beta}\left(\sigma_{x}^{(j)}\right) a_{x}^{(j)}\right) \Omega=0 .
$$

Equations (40) and (44) tell us the following identity:

$$
\tilde{\psi}\left(Q_{1} L_{j}\left(Q_{2}\right)\right)=\tilde{\psi}\left(L_{j}\left(Q_{1}\right) Q_{2}\right) .
$$

The reversibility of $\tilde{\psi}$ for $S_{t}$ follows from this.

The next thing we explain is the ergodicity criterion of the Markov semigroup $S_{t}$. We introduce the free motion for the infinite temperature state,

$$
L_{j}^{F}(Q)=\operatorname{tr}_{j}\left(P_{j} Q P_{j}\right)-Q,
$$

where $\operatorname{tr}_{j}$ is the normalized partial trace on the site $j, \sigma_{0}=1$,

$$
\operatorname{tr}_{j}(Q)=\frac{1}{16} \sum_{\alpha, \beta=0, x, y, z} \sigma_{\alpha}^{(j)} \otimes \sigma_{\beta}^{(j)} Q \alpha \otimes \sigma_{\beta}^{(j)}
$$

and

$$
P_{j}=\frac{1}{2}\left(1+\sigma_{z}^{(j)} \otimes \sigma_{z}^{(j)}\right)\left(1+\sigma_{x}^{(j)} \otimes \sigma_{x}^{(j)}\right) .
$$

If the inverse temperature $\beta$ is zero, we can show

$$
L_{j}^{F}(Q)=L_{j}(Q) \text {. }
$$

Then set

$$
L^{F}(Q)=\sum_{j \in \mathbf{Z}^{\mathbf{d}}} L_{j}^{F}(Q)
$$

It is easy to see

$$
\left.\tilde{\delta}_{(\alpha, \beta, j)}(L){ }_{j}^{F}(Q)\right)=-\tilde{\delta}_{(\alpha, \beta, j)}(Q) .
$$

We have the following estimate. (See [7].)

$$
\left\|\left|\exp \left(t L^{F}\right)(Q)\right|\right\| \leqq e^{-t}\||Q|\| .
$$

We next consider

$$
A_{j}(Q)=L_{j}(Q)-L_{j}^{F}(Q)
$$


Proposition 2. Let $\left\{\gamma_{j i}^{(l)} \mid l \in \mathbf{Z}^{\mathbf{d}}, j, i \in \tilde{X}\right\}$ be a non-negative matrix satisfying

$$
\left\|\left(\tilde{\delta}_{j} A_{l}-A_{l} \tilde{\delta}_{j}\right)(Q)\right\| \leqq \sum_{i} \gamma_{j i}^{(k)}\left\|\tilde{\delta}_{i}(Q)\right\|
$$

Set

$$
\gamma=\sup _{i \in \tilde{X}}\left(\sum_{j} \sum_{l} \gamma_{j i}^{(l)}\right)
$$

If $\gamma<l$, we have

$$
\left\|\left|S_{t}(Q)\right|\right\| \leqq e^{-t(1-\gamma)}\||Q|\|
$$

for any $Q$ in $C^{1}(\tilde{\mathscr{A}})$.

As a consequence, $S_{t}$ has the unique invariant state and the Gibbs state is unique.

Remark 1. (i) It is possible to show that Assumption 1 implies the finiteness of $\gamma$. (ii) By the estimate obtained in the next section, $\gamma$ is the order of $\beta$ (the inverse temperature) for small $\beta$. So the above proposition is valid at least for high temperatures. At the moment, we have no other example of $\gamma<1$.

Proof of Proposition 2. The proof of Proposition 2 is more or less standard in classical spin models. The proof of the page 184 of [8] works for our noncommutative case. Here we give its sketch.

Set

$$
L^{j}=L-L_{j}^{F}, \quad S_{t}^{(j)}=\exp \left(t L^{j}\right) .
$$

Then

$$
\frac{d}{d s} e^{s} S_{t-s}^{(j)} \tilde{\delta}_{k} S_{s}(Q)=e^{s} S_{t-s}^{(j)}\left(\widetilde{\delta_{k}} A_{j}-A_{j} \widetilde{\delta_{k}}\right) S_{s}(Q)
$$

if $k=(j, \tilde{\alpha})$.

By integrating $s$ from 0 to $t$, we obtain

$$
\left\|e^{t} \tilde{\delta}_{k} S_{t}(Q)\right\| \leqq\left\|\tilde{\delta}_{k}(Q)\right\| \int_{0}^{t} e^{s}\left\|\left(\sum_{l}\left(\widetilde{\delta_{k}} A_{l}-A_{l} \widetilde{\delta_{k}}\right) S_{s}\right)(Q)\right\| d s
$$

Taking the sum in $k$, we arrive at

$$
e^{t}\left\|\left|S_{t}(Q)\right|\right\| \leqq|\|(Q)\||+\gamma \int_{0}^{t} e^{s}\left\|\left|S_{s}(Q)\right|\right\| d s .
$$

This implies (56).

\section{Analysis on Quantum Spin Algebra}

The aim of this section is to verify Assumption 1 for the case of the high temperature Gibbs state. So we consider the time evolution of quantum observables and its analytic extension. Our estimate is far from best possible, but its proof is very simple. 
We introduce some notations. For a pair of finite subsets A and B of the lattice $\mathbf{Z}^{\text {d, we set }}$

$$
\begin{gathered}
C=A \times\{z\} \cup B \times\{x\} \in X, \\
\sigma(C)=\prod_{k \in A} \sigma_{z}^{(k)} \prod_{j \in B} \sigma_{x}^{(j)} . \\
\operatorname{supp} C=A \cup B .
\end{gathered}
$$

For $j$ in $\mathbf{Z}^{\mathbf{d}}$, we set

$$
C+j=\{A+j\} \times\{z\} \cup\{B+j\} \times\{x\} \in X,
$$

so

$$
\tau_{j}(\sigma(C))=\sigma(C+j)
$$

The Hamiltonian is now

$$
H=\sum_{j} \tau_{\overline{(h)}}, h=\sum_{C} h(C) \sigma(C) .
$$

Proposition 3. Let $H$ be the translationally invariant Hamiltonian (19) satisfying the decay condition of (23) with $\varepsilon=\log (1+2 \lambda)(\lambda>0)$. Let $Q$ be an element of $C_{\lambda}^{\infty}(\mathscr{A})$

Then, the time evolution $\alpha_{t}(Q)$ of $Q$ as a function of $t$ can be extended to a holomorphic function $\alpha_{z}(Q)$ on the disc $\left\{z \in C|z|<R^{-1}\right\}$, where

$$
R=\frac{2}{\lambda}\left(\sum_{C}|h(C) \| C|(1+2 \lambda)^{|C|}\right)
$$

and

$$
\lambda^{n}\left\|\left|\alpha_{z}(Q)\right|\right\|_{n} \leqq(1-|z| R)^{-n}\left(\sup _{k} \lambda^{k}\||Q|\|_{k}\right) .
$$

Proposition 3 shows if $\lambda>1$ and $\beta$ is small, Assumption 1 is valid.

Proposition 3 is an immediate consequence from the following estimate.

\section{Lemma 2.}

$$
\lambda^{n}\||[H, Q]|\|_{n} \leqq R(n+1) \sup _{1 \leqq k \leqq n+1} \quad \lambda^{k}\||Q|\|_{k} .
$$

Proof. In the case of $n=0$, we have

$$
\|[H, Q]\| \leqq \sum_{j}\left|h_{C}\right|\|[\sigma(C+j), Q]\| \leqq 2 \sum_{j}\left|h_{C}\right| \sum_{b \in C+j}\left\|\delta_{b}(Q)\right\|,
$$

where we used the following inequality:

$$
\|[\sigma(C), Q]\| \leqq 2 \sum_{b \in C}\left\|\delta_{b}(Q)\right\| .
$$

In the above, consider a fixed $b$ in $X$ and the number of $j$ which contributes to the sum is $|C|$, so we get

$$
\sum_{j} \sum_{b \in C+j}\left|\delta_{b}(Q)\right|=|C|\||Q|\|_{1}
$$


As a consequence, we obtain

$$
|\|[H, Q]\||_{0} \leqq 2 \sum_{C} \mid h_{C}\|C\|\|Q\|_{1} .
$$
First,

The case of $n$ larger than or equal to 1 can be handled in the following way.

$$
\left\|\delta_{b_{1}} \delta_{b_{2}} \ldots \delta_{b_{n}}([H, Q])\right\| \leqq \sum_{j} \sum_{C}|h(C)|\left\|\delta_{b_{1}} \delta_{b_{2}} \ldots \delta_{b_{n}}([\sigma(C+j), Q])\right\| .
$$

If $b_{i}=(k, \alpha), k \notin \operatorname{supp}(C+j)$,

$$
\left\|\delta_{b_{1}} \delta_{b_{2}} \ldots \delta_{b_{n}}([\sigma(C+j), Q])\right\|=\left\|\delta_{b_{1}} \ldots \delta_{b_{t-1}} \delta_{b_{t+1}} \ldots \delta_{b_{n}}\left(\left[\sigma(C+j), \delta_{b_{1}}(Q)\right]\right)\right\|
$$

If $b_{i}=(k, \alpha)$ and $k$ belongs to $\operatorname{supp}(C+j)$,

$$
\left\|\delta_{b_{1}} \delta_{b_{2}} \ldots \delta_{b_{n}}([\sigma(C+j), Q])\right\| \leqq\left\|\delta_{b_{1}} \ldots \delta_{b_{i-1}} \delta_{b_{i+1}} \ldots \delta_{b_{n}}([\sigma(C+j), Q])\right\| .
$$

where we used

$$
\begin{aligned}
\delta_{b} \delta_{b^{\prime}} & =\delta_{b^{\prime}} \delta_{b}, \\
\delta_{b}\left(\sigma_{k}^{(\alpha)} Q \sigma_{k}^{(\alpha)}\right. & =\sigma_{k}^{(\alpha)} \delta_{b}(Q) \sigma_{k}^{(\alpha)}
\end{aligned}
$$

for any $b$ in $X, k$ in $\mathbf{Z}^{\mathbf{d}}$ and $\alpha=x, y, z$.

So if $b_{l}=\left(\alpha_{l}, i_{l}\right)$ and $i_{l} \in \operatorname{supp}(C+j)(1 \leqq l \leqq m) i_{l} \notin \operatorname{supp}(C+j)(m+1 \leqq l \leqq n)$,

$$
\left\|\delta_{b_{1}} \ldots \delta_{b_{n}}([\sigma(C+j), Q])\right\| \leqq\left\|\left[\sigma(C+j), \delta_{b_{m+1}} \ldots \delta_{b_{n}}(Q)\right]\right\| \text {. }
$$

We now obtain

$$
\begin{aligned}
& \||[\sigma(C+j), Q]|\|_{n} \\
& \leqq \sum_{k=1}^{\min (n,|C|)}\left\{|C| C_{n-k} 2^{n-k} \sum_{A=\left\{b_{1} \ldots b_{k}\right\} \in F_{k}, A \cap C+j=\emptyset}\left\|\left[\sigma(C+j), \delta_{b_{1}} \ldots \delta_{b_{k}}(Q)\right]\right\|\right\},
\end{aligned}
$$

where the factor $2^{n-k}$ comes from the assignment of $x$ and $z$ components to points in $C+j$. Thus

$$
\begin{aligned}
& \sum_{j}\||[\sigma(C+j), Q]|\|_{n} \\
& \quad \leqq \sum_{j} \sum_{k=1}^{|C|}{ }_{|C|} C_{n-k} \sum_{A=\left\{b_{1} \ldots b_{k}\right\} \in F_{k}, A \cup C+j=\emptyset}\left\|\left[\sigma(C+j), \delta_{b_{1}} \ldots \delta_{b_{k}}(Q)\right]\right\| \\
& \quad \leqq 2 \sum_{k=1}^{|C|}|C| C_{n-k} \sum_{j} \sum_{b \in C+j} \sum_{A \in F_{k} b \notin A}\left\|\delta_{b} \delta_{A}(Q)\right\| \\
& \quad \leqq 2 \sum_{k=1}^{|C|}|C| C_{n-k}|C|\{n-k+1\} 2^{n-k}\||Q|\|_{k+1}, \\
& \lambda^{n}\||[H, Q]|\|_{n} \\
& \quad \leqq 2 \frac{1}{\lambda}\left(\sum_{C}|C||h(C)| \sum_{k=0}^{|C|}{ }_{|\leq|} C_{k} \lambda^{k} 2^{k}\right)\left(\sup _{1 \leqq l \leqq n+1}(n-l+1) \lambda^{l}\||Q|\|_{l}\right) .
\end{aligned}
$$

This last estimate implies our claim. 
Proof of Proposition 3. We just expand $\alpha_{z}(Q)$ by $z$. Using the lemma just proved above, we get

$$
\begin{aligned}
\lambda^{l}\left\|\left|\alpha_{z}(Q)\right|\right\| & \leqq \sum_{n=0}^{\infty} \frac{|z|^{n}}{n !} \frac{(l+n) !}{l !}\left(\sup _{0 \leqq k<\infty} \lambda^{l}\||Q|\|_{l}\right) \\
& \leqq\left.\frac{1}{l !} \sum_{n=0}^{\infty}\left(\frac{d}{d x}\right)^{l} x^{l+n}\right|_{x=|z| R}\left(\sup _{0 \leqq k<\infty} \lambda^{l}\||Q|\|_{l}\right) \\
& \leqq\left.\frac{1}{l !}\left(\frac{d}{d x}\right)^{l} \frac{1}{1-x}\right|_{x=|z| R}\left(\sup _{0 \leqq k<\infty} \lambda^{l}\||Q|\|_{l}\right) \\
& \leqq \frac{1}{(1-|z| R)^{l}}\left(\sup _{0 \leqq k<\infty} \lambda^{l}\||Q|\|_{l}\right) .
\end{aligned}
$$

Acknowledgement. This work was done while the author was visiting the Institute for Theoretical Physics, University of Leuven as a research fellow of Canon Foundation in Europe. The author acknowledges the financial support from Canon Foundation and the warm hospitality of the Institute for Theoretical Physics, University of Leuven.

\section{References}

1. Araki, H., Ion, P.: On equivalence of KMS and Gibbs conditions for states of quantum lattice systems. Commun. Math. Phys. 35, 1-12 (1974)

2. Bratteli, O., Robinson, D.: Operator Algebras and Quantum Statistical Mechanics. Vol. I and II, Berlin, Heidelberg, New York: Springer

3. Bratteli, O., Robinson, D.: Unbounded derivation of von Neumann algebras. Ann. Inst. Henri Poincaré 25, 159-164 (1976)

4. Dobrushin, R.L.: Theory Probab. Its Appl. 13, 13, 197-224 (1968)

5. Dobrushin, R.L.: Shlosman, B.: Constructive criterion for the uniqueness of Gibbs field. In: Statistical Physics and Dynamical Systems. Rigorous Results, Basel, Birkhäuser: 1985, pp. $347-370$

6. Kossakowski, A., Frigerio, A., Gorini, V., Verni, M.: Detailed balance and KMS conditions. Commun. Math. Phys. 57, 97-110 (1977)

7. Matsui, T.: Markov semigroups on UHF algebras. To appear

8. Stroock, D.W., Zegalinski B.: The logarithmic Sobolev inequality for discrete spin systems on a lattice. Commun. Math. Phys. 149, 175-193 (1992)

Communicated by $\mathrm{H}$. Araki 